\title{
Association of presence/absence and on/off patterns of Helicobacter pylori oipA gene with peptic ulcer disease and gastric cancer risks: a meta-analysis
}

Jingwei Liư ${ }^{\dagger}$, Caiyun $\mathrm{He}^{\dagger}$, Moye Chen, Zhenning Wang, Chengzhong Xing ${ }^{*}$ and Yuan Yuan ${ }^{*}$

\begin{abstract}
Background: There are increasing studies examining the relationship between the status of H. pylori oipA gene and peptic ulcer disease (PUD) and gastric cancer (GC) but the results turn out to be controversial. We attempted to clarify whether oipA gene status is linked with PUD and/or GC risks.

Methods: A systematically literature search was performed through four electronic databases. According to the specific inclusion and exclusion criteria, seven articles were ultimately available for the meta-analysis of oipA presence/absence with PUD and GC, and eleven articles were included for the meta-analysis of oipA on/off status with PUD and GC.

Results: For the on/off functional status analysis of oipA gene, the "on" status showed significant associations with increased risks of PUD $(\mathrm{OR}=3.97,95 \% \mathrm{Cl}: 2.89,5.45 ; \mathrm{P}<0.001)$ and $\mathrm{GC}(\mathrm{OR}=2.43,95 \% \mathrm{Cl}: 1.45,4.07 ; \mathrm{P}=0.001)$ compared with gastritis and functional dyspepsia controls. Results of the homogeneity test indicated different effects of oipA "on" status on PUD risk between children and adult subgroups and on GC risk between PCR-sequencing and immunoblot subgroups. For the presence/absence analysis of oipA gene, we found null association of the presence of oipA gene with the risks of PUD (OR $=1.93,95 \% \mathrm{Cl}: 0.60,6.25 ; \mathrm{P}=0.278)$ and $\mathrm{GC}(\mathrm{OR}=2.09,95 \% \mathrm{Cl}: 0.51$, 8.66; $P=0.308)$ compared with gastritis and functional dyspepsia controls.

Conclusions: To be concluded, when oipA exists, the functional "on" status of this gene showed association with increased risks for PUD and GC compared with gastritis and FD controls. However, merely investigating the presence/absence of oipA would overlook the importance of its functional on/off status and would not be reliable to predict risks of PUD and GC. Further large-scale and well-designed studies concerning on/off status of oipA are required to confirm our meta-analysis results.
\end{abstract}

Keywords: Helicobacter pylori, OipA, Peptic ulcer disease, Gastric cancer

\section{Background}

Helicobacter pylori (H. pylori) infection is the most important risk factor for the development of peptic ulcer disease (PUD) and gastric cancer (GC) [1]. Nearly half of the population worldwide harbors $H$. pylori, however, both GC and PUD occur in only a small portion of those

\footnotetext{
*Correspondence: xcz1966@126.com; yyuan@mail.cmu.edu.cn

${ }^{\dagger}$ Equal contributors

Tumor Etiology and Screening Department of Cancer Institute and General Surgery, the First Affiliated Hospital of China Medical University, and Key Laboratory of Cancer Etiology and Prevention (China Medical University), Liaoning Provincial Education Department, Shenyang 110001, China
}

carrying $H$. pylori [2]. Difference in the consequence of $H$. pylori infection could be at least partially explained by the high variability of colonizing $H$. pylori strains and host response to this microbe [3]. In addition to the host genetic susceptibility, sequence diversity of $H$. pylori virulence factor gene may affect the ability of these bacteria to colonize, persist, and/or induce severe diseases, and thus enable us to predict the consequences of their carriers [4].

H. pylori possesses various genes, some of which are virulence genes associated with gastroduodenal diseases. 
To date, several well-described virulence factors of $H$. pylori including CagA (cytotoxin-associated gene A product) and VacA (vacuolating cytotoxin A) have been linked to severe gastroduodenal diseases such as PUD and GC [5]. Apart from CagA and VacA, other virulence factors were discovered successively. A study reported by Yamaoka Y. et al. in 2000 and subsequent studies provided evidence that OipA (outer inflammatory protein A) is another important virulence factor in relation to the risks of PUD and GC [6]. The oipA gene (also known as HP0638) encoding for OipA protein is regulated by a switch (i.e. 'on' or 'off' patterns) by changing the number of $\mathrm{CT}$ dinucleotide repeats in the signal-peptide coding region of this gene. Yamaoka Y. et al's report also unraveled that the on/off functional status of oipA is accommodated by slipped strand mispairing mechanism [4]. When there is 6,9 , $(1+3),(2+3),(1+2),(1+1+1),(1+1+2)$ or other CT dinucleotide repeats that keep the peptide in frame, oipA gene is "on" status. Otherwise, the status is "off", which is nonfunctional oipA gene [7]. This OipA protein appeared to be particularly important in inducing interleukin 8 (IL-8) secretion and facilitating the bacteria's colonization in stomach [1].

Since the discovery of oipA in 2000 [6], the relationship between the status of oip $A$ gene and risks of PUD and GC is of special interest. Two kinds of studies in relation to oipA gene were emerging, some of which investigated the presence/absence of oipA [8-14] while the others examined the functional on/off status of oipA [6,15-24]. However, the results of these studies turn out to be controversial mainly due to relatively small sample size. So far, no meta-analysis has been provided to evaluate the risks of PUD and GC in relation to oipA gene presence/absence or its on/off status. In the present study, aiming at elucidating the role of oipA genetic diversity in modulating the risks of PUD and/or GC, we performed a meta-analysis to investigate the association between the presence/absence and on/off status of oipA gene and PUD and GC risks.

\section{Methods}

\section{Identification and eligibility of relevant studies}

We systematically search the literatures of electronic databases including PubMed, Web of Science, Chinese National Knowledge Infrastructure (CNKI) and Wanfang database using the search terms of "OipA", "outer inflammatory protein A" and "HP0638" in English databases and their corresponding Chinese terms in Chinese databases. When overlapping data exists, only the largest and latest study was selected for this meta-analysis. We contacted the author for specific raw data if the data presented in the article were not clear. The last search date was December 6, 2012.
Studies included in the present meta-analysis must meet the inclusion criteria as follows: observational studies concerning the association between the presence/ absence (also defined as positive/negative) or on/off status of oipA gene and PUD or GC with control group of gastritis or functional dyspepsia (FD); studies published in English or Chinese; studies with $H$. pylori-positive cases and controls; studies with sufficient raw data for estimating odds ratios (OR) and their 95\% confidence interval (CI). The main reasons for exclusion were reviews; animal experiments; case series; duplicate publications; no raw data after contacting the author; studies not in English or Chinese; and conference proceedings.

\section{Data extraction}

Two authors (Jingwei Liu and Caiyun He) extracted the data from the inclusive studies independently. The conflict was resolved after discussion and consensus was finally reached on all of the extracted data. The following information was extracted from each study: first author, year of publication, ethnicity of the population, numbers of cases and controls, detection methods for oipA gene and $H$. pylori infection, primers for polymerase chain reaction (PCR), source of $H$. pylori isolates, adult or children of the study subjects, and disease diagnosis for control group.

\section{Evaluation of the validity of the included studies}

We used the eight-item Newcastle-Ottawa scale (NOS) to assess the validity of the included studies [25], in which a study could be awarded a maximum of nine stars on items related to the selection (four stars), the comparability (two stars) and the exposure (three stars). The NOS scores of 1-3, 4-6, 7-9 were considered as low, intermediate and high quality, respectively.

\section{Statistical analysis}

The statistical analysis was carried out by Stata software (Version 11.0; StataCorp, College Station, TX). The strength of association between the presence/absence or on/off status of oipA gene and PUD or GC was assessed by OR and their $95 \%$ CI. P value $<0.05$ was considered as statistically significant. Heterogeneity was measured by using $\mathrm{Q}$ statistic $(\mathrm{P}<0.10$ indicates significant heterogeneity between studies) and I-squared $\left(\mathrm{I}^{2}\right)$ value [26]. A fixed-effects model using Mantel-Haenszel method [27] was applied to calculate the pooled ORs when heterogeneity between studies was not significant. Otherwise, a random-effects model using DerSimonian and Laird method [28] was performed. Sensitivity analysis was performed to explore heterogeneity when significant heterogeneity was indicated. Subgroup analyses were performed to explore the effects of geographical region, GU or DU, adult or children, and oipA gene detection 
method. To compare the effect of oipA gene status on the risks of PUD and GC among different subgroups, the Breslow-Day test was used to assess the homogeneity of stratum-specific ORs across different subgroups. For Breslow-Day test, statistical significance was noted as $\mathrm{P} \leq 0.10$. In addition, publication bias were evaluated qualitatively by performing funnel plots and assessed quantitatively by Begg's test [29] and Egger's test [30], respectively. $\mathrm{P}$ value $<0.1$ for Begg's and Egger's tests indicates significant publication bias.

\section{Results}

\section{Study characteristics}

This meta-analysis was organized according to the PRISMA statement (Additional file 1). A total of 194 potentially relevant records were found through four databases after duplicates removal; 176 articles were further excluded for main reasons of no relevance, in vitro or animal experiments, reviews, meeting abstract, data covered by other studies, and no raw data. Finally, 18 full-text articles with eligibility were included in this meta-analysis [6,8-24]. The flow chart of article selection was presented in Figure 1.

The main characteristics of the studies included in this meta-analysis were summarized in Table 1 and additional information of primary studies was showed in Additional file 2: Table S1 and Table S2. All the included studies were case-control designed. For meta-analysis of the on/off status of oipA gene, 11 articles met the inclusion criteria
[6,15-24]. One article reported by Yamaoka, Y. in Japanese in 2000 found that the oipA gene status was $100 \%$ "on" in the cases and controls, which could not be analyzed in this meta-analysis. Therefore, 10 articles were finally eligible for the meta-analysis of the oipA on/off status. Among them, the functional on/off status of oipA was determined by the PCR-based sequencing with one exception of Yamaoka, Y et al's study [21] in which immunoblotting was used to directly evaluate the protein expression of oipA; data were from three geographical regions including Asia, Europe and America; and one article [20] involved subjects of two different age groups (adults and children). For meta-analysis of the presence/absence of oipA gene, seven articles were ultimately eligible [8-14]. The presence/absence of oipA gene in the included articles was all detected by the PCR-based electrophoresis which could not reflect the functional status of oipA. Six articles were about Asian populations except one article involving Tunisian [9]. One article reported by Dabiri, $\mathrm{H}$. et al. [11] involved study populations from three Asian countries. Considering a potential role of geographic location in altering the oipA gene status, data from different regions were treated as separate studies in the subgroup analysis. Data from different regions and different age groups were treated as separate studies in the subgroup analysis.

The NOS results indicated that all the included studies were at an intermediate level of quality with scores ranging

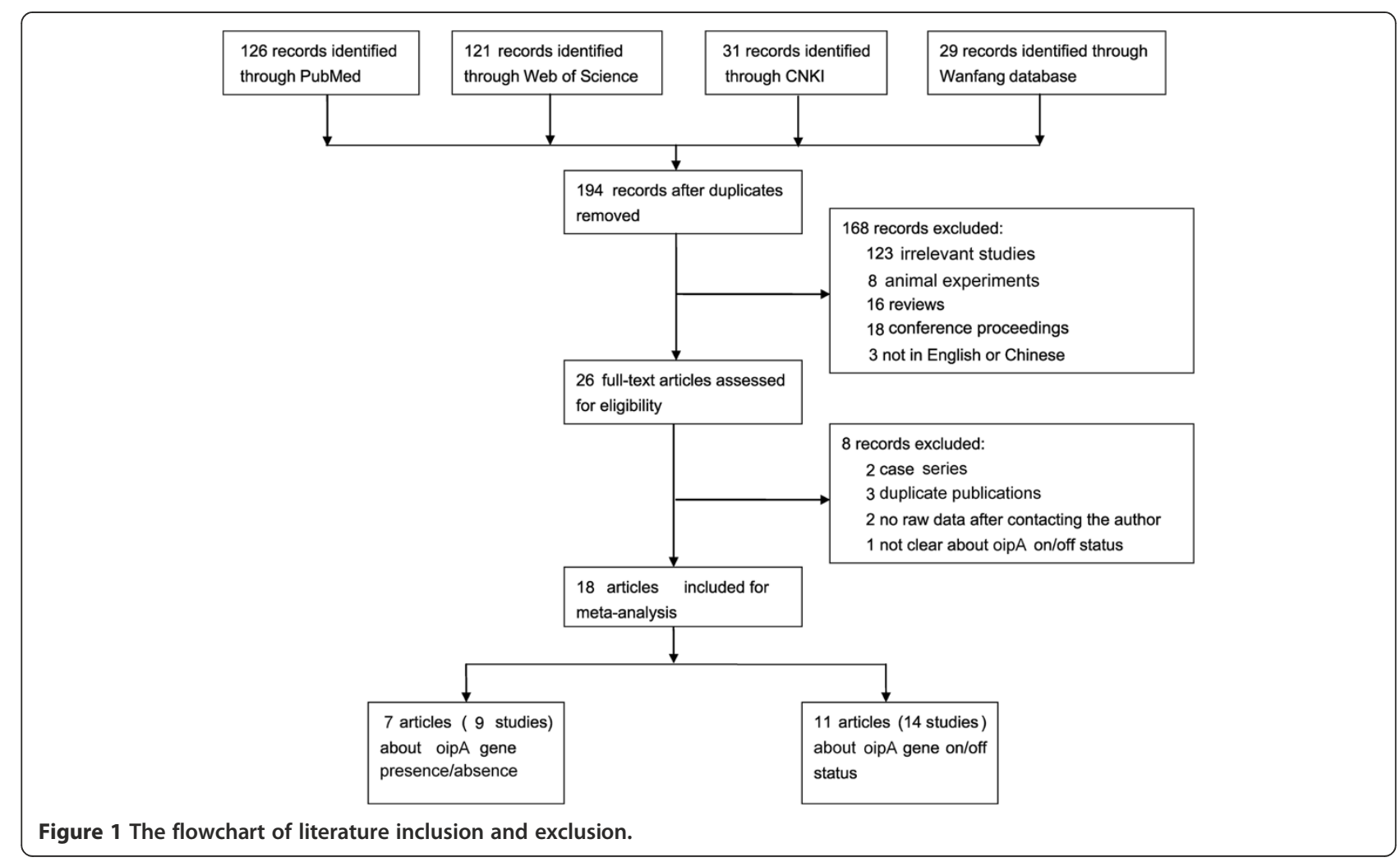


Table 1 Characteristics of selected studies for oipA gene on/off and presence/absence status analysis

\begin{tabular}{|c|c|c|c|c|c|c|c|c|}
\hline Author & Ethnicity & Year & Region & $\begin{array}{c}\text { gastritis or FD } \\
\text { oipA+/total } \\
(\%)^{d}\end{array}$ & $\begin{array}{c}\text { PUD } \\
\text { oipA+/tota } \\
(\%)^{d}\end{array}$ & $\begin{array}{c}\mathrm{GU} \\
\text { oipA+/total } \\
(\%)^{d}\end{array}$ & $\begin{array}{c}\text { DU } \\
\text { oipA+/total } \\
\text { cases }(\%)^{d}\end{array}$ & $\begin{array}{c}\text { GC } \\
\text { oipA+/total } \\
{\text { cases }(\%)^{d}}^{\text {d }}\end{array}$ \\
\hline \multicolumn{9}{|c|}{ For oipA functional status (on/off) studies } \\
\hline Markovska, R. & Bulgarian & 2011 & Europe & 23/35(65.7\%) & $33 / 34(97.1 \%)$ & I & I & I \\
\hline Oleastro, M. & Portuguese & 2010 & Europe & 18/60(30.0\%) & 40/57(70.2\%) & / & / & / \\
\hline Schmidt, H. M. & Chinese & 2010 & Asia & $45 / 52(86.5 \%)$ & 14/16(87.5\%) & / & $14 / 16(87.5 \%)$ & 19/22(86.4\%) \\
\hline Chiarini, A. & Italian & 2009 & Europe & 17/21(81.0\%) & 7/10(70.0\%) & / & / & / \\
\hline $\mathrm{LI}, \mathrm{N}$. & Chinese & 2009 & Asia & 66/86(76.7\%) & $12 / 12(100.0 \%)$ & $12 / 12(100.0 \%)$ & / & $2 / 2(100.0 \%)$ \\
\hline \multirow[t]{2}{*}{ Oleastro, M. } & Portuguese $^{a}$ & 2008 & Europe & $28 / 56(50.0 \%)$ & $34 / 50(68.0 \%)$ & / & / & / \\
\hline & Portuguese $^{b}$ & 2008 & Europe & 17/53(32.1\%) & 26/31(83.9\%) & 1 & 1 & 1 \\
\hline \multirow[t]{2}{*}{ Yamaoka, Y. } & Colombian & 2006 & America & $24 / 40(60.0 \%)$ & $36 / 40(90.0 \%)$ & I & $36 / 40(90.0 \%)$ & $36 / 40(90.0 \%)$ \\
\hline & American & 2006 & America & $25 / 40(62.5 \%)$ & $34 / 40(85.0 \%)$ & 1 & $34 / 40(85.0 \%)$ & 1 \\
\hline de Jonge, R. & Dutch & 2004 & Europe & 18/29(62.1\%) & 39/49(79.6\%) & 16/21(76.2\%) & 23/28(82.1\%) & 8/9(88.9\%) \\
\hline Zambon, C. F. & Itilian & 2003 & Europe & 15/31(48.4\%) & 14/19(73.7\%) & I & I & / \\
\hline \multirow[t]{2}{*}{ Yamaoka, Y. } & American & 2002 & America & 25/40(62.5\%) & $37 / 41(90.2 \%)$ & / & $37 / 41(90.2 \%)$ & $20 / 30(66.7 \%)$ \\
\hline & Colombian & 2002 & America & $26 / 40(65.0 \%)$ & $36 / 40(90.0 \%)$ & 1 & $36 / 40(90.0 \%)$ & $34 / 41(82.9 \%)$ \\
\hline Yamaoka, Y. & Japanese & 2000 & Asia & 40/40(100.0\%) & $40 / 40(100.0 \%)$ & I & 40/40(100.0\%) & / \\
\hline \multicolumn{9}{|c|}{ For oipA gene (presence/absence) studies } \\
\hline Ji, C. W. & Chinese & 2011 & Asia & $26 / 46(56.5 \%)$ & $45 / 58(77.6 \%)$ & $45 / 58(77.6 \%)$ & / & $33 / 43(76.7 \%)$ \\
\hline Ben, M. K. & Tunisian & 2010 & Africa & 186/195(95.4\%) & 63/78(80.8\%) & / & / & / \\
\hline Xie, J. & Chinese & 2010 & Asia & 16/51(31.4\%) & 12/39(30.8\%) & 6/19(31.6\%) & $6 / 20(30.0 \%)$ & 19/27(70.4\%) \\
\hline \multirow[t]{3}{*}{ Dabiri, $\mathrm{H}$. } & Persians & 2009 & Asia & $36 / 57(63.2 \%)$ & $2 / 13(15.4 \%)$ & I & I & $0 / 4(0.0 \%)$ \\
\hline & Turkish & 2009 & Asia & $7 / 21(33.3 \%)$ & $3 / 5(60.0 \%)$ & I & / & $1 / 7(14.3 \%)$ \\
\hline & Kurds, etc. $^{c}$ & 2009 & Asia & $8 / 13(61.5 \%)$ & $4 / 4(100.0 \%)$ & / & / & / \\
\hline Zhou, M. & Chinese & 2009 & Asia & $21 / 40(52.5 \%)$ & $37 / 40(92.5 \%)$ & I & $37 / 40(92.5 \%)$ & 18/20(90.0\%) \\
\hline Salih, B. A. & Turkish & 2007 & Asia & 17/21(81.0\%) & 13/14(92.9\%) & 1 & I & I \\
\hline Zhang, J. & Chinese & 2004 & Asia & 11/42(26.2\%) & 16/16(100.0\%) & 16/16(100.0\%) & / & / \\
\hline
\end{tabular}

${ }^{a}$, for adult study population; ${ }^{b}$, for children study population; ${ }^{c}$, Kurds, Lurs, Afghanis, Arabs; ${ }^{\mathrm{d}}$, for oipA gene presence/absence studies, oipA(+) means oipA gene positive, while for oipA functional status on/off studies, oipA(+) means oipA gene "on" status.

Abbreviations: FD functional dyspepsia, PUD peptic ulcer disease, GU gastric ulcer, DU duodenal ulcer, GC gastric cancer.

from 4 to 6 , because some studies did not provide specific selection criteria of control group and most studies did not fully consider the control factor for the comparability of cases and controls such as age and sex. Detailed results for NOS quality assessment were summarized in Additional file 2: Table S3.

\section{Association between oipA on/off status and PUD and GC} In the pooled estimate for PUD, we observed that oipA "on" status was significant associated with an increased its overall risk compared with gastritis and FD controls (OR = 3.97, 95\% CI: 2.89, 5.45; $\mathrm{P}<0.001$, Table 2 and Figure 2). Meanwhile, no significant heterogeneity existed among studies $\left(\mathrm{I}^{2}=22.30 \%, \mathrm{P}=0.238\right)$. Subgroup analysis was then performed to investigate the effects of geographical region, detection method of oipA gene and adult or children of the studied population. Consistent increased risks for PUD development were found in all subgroup analyses, with ORs ranging from 2.42 to 7.03 . However, the result in
Asia subgroup did not reach statistical significance $(\mathrm{P}=$ 0.208). Result of Breslow-Day test indicated no significantly different effects of oipA "on" status on PUD risk among Asia/Europe/America subgroups $(\mathrm{P}=0.681$, Table 2). The OR for PUD risk was higher in children subgroup than that in adult subgroup, and meanwhile, the effect difference reached statistical significance (for Breslow-Day test: $\mathrm{P}=$ 0.014). For DU and GU separately, we observed that oipA "on" status was significant associated with an increased risk of DU compared with gastritis and FD controls (OR = 3.83, 95\% CI: 2.32, 6.34; $\mathrm{P}<0.001$ ), but the association between oipA "on" status and GU risk did not reach statistical significance $(\mathrm{OR}=2.85,95 \% \mathrm{CI}: 0.94,8.69 ; \mathrm{P}=0.065)$.

In the pooled estimate for GC, the oipA "on" status was significantly associated with its overall risk compared with gastritis and FD controls (OR $=2.43,95 \% \mathrm{CI}: 1.45$, 4.07; $\mathrm{P}=0.001$, Table 2 and Figure 3). No significant heterogeneity existed among studies $\left(\mathrm{I}^{2}=16.80 \%, \mathrm{P}=0.380\right)$. In the subgroup analysis according to geographical 
Table 2 Meta-analysis results for association between oipA on/off and presence/absence status and PUD and GC

\begin{tabular}{|c|c|c|c|c|c|c|c|}
\hline Variable & No. of studies & No. of cases/controls & OR $(95 \% \mathrm{Cl})$ & $P$ value & $I^{2}$ & $P_{\text {het }}{ }^{a}$ & $P_{B-D \text { test }}{ }^{b}$ \\
\hline \multicolumn{8}{|l|}{ For oipA on/off studies } \\
\hline \multicolumn{8}{|l|}{ PUD vs. gastritis/FD } \\
\hline All & 10 & $439 / 583$ & $3.97(2.89,5.45)$ & $<0.001$ & $22.30 \%$ & 0.238 & \\
\hline Region & & & & & & & 0.681 \\
\hline Europe & 7 & $250 / 285$ & $3.75(2.54,5.53)$ & $<0.001$ & $57.20 \%$ & 0.029 & \\
\hline Asia & 2 & $28 / 138$ & $2.42(0.61,9.55)$ & 0.208 & $32.90 \%$ & 0.222 & \\
\hline America & 4 & $161 / 160$ & $4.77(2.65,8.56)$ & $<0.001$ & $0.00 \%$ & 0.902 & \\
\hline Detection technique & & & & & & & 0.376 \\
\hline PCR based-sequencing & 11 & $359 / 503$ & $3.83(2.71,5.40)$ & $<0.001$ & $41.10 \%$ & 0.075 & \\
\hline Immunoblot & 2 & $80 / 80$ & $4.42(1.98,9.83)$ & $<0.001$ & $0.00 \%$ & 0.492 & \\
\hline Age & & & & & & & 0.014 \\
\hline Adult & 11 & $351 / 470$ & $3.26(2.26,4.69)$ & $<0.001$ & $16.90 \%$ & 0.282 & \\
\hline Children & 2 & $88 / 113$ & $7.03(3.71,13.34)$ & $<0.001$ & $0.00 \%$ & 0.319 & \\
\hline GU vs. gastritis/FD & 2 & $33 / 115$ & $2.85(0.94,8.69)$ & 0.065 & $0.00 \%$ & 0.368 & \\
\hline DU vs. gastritis/FD & 6 & $205 / 241$ & $3.832 .32,6.34)$ & $<0.001$ & $0.00 \%$ & 0.628 & \\
\hline \multicolumn{8}{|l|}{ GC vs. gastritis/FD } \\
\hline All & 5 & $144 / 287$ & $2.43(1.45,4.07)$ & 0.001 & $16.80 \%$ & 0.308 & \\
\hline Region & & & & & & & 0.697 \\
\hline Europe & 1 & $9 / 29$ & $4.89(0.54,44.57)$ & 0.159 & / & / & \\
\hline Asia & 2 & $24 / 138$ & $1.08(0.29,3.99)$ & 0.909 & $0.00 \%$ & 0.796 & \\
\hline America & 3 & $111 / 120$ & $2.47(1.36,4.51)$ & 0.003 & $51.30 \%$ & 0.128 & \\
\hline Detection technique & & & & & & & 0.034 \\
\hline PCR based-sequencing & 5 & $104 / 247$ & $1.75(0.96,3.18)$ & 0.066 & $0.00 \%$ & 0.634 & \\
\hline Immunoblot & 1 & $40 / 40$ & $6.00(1.79,20.15)$ & 0.004 & l & / & \\
\hline \multicolumn{8}{|c|}{ For oipA presence/absence studies } \\
\hline \multicolumn{8}{|l|}{ PUD vs. gastritis/FD } \\
\hline All & 7 & $267 / 486$ & $1.93(0.60,6.25)$ & 0.278 & $85.70 \%$ & $<0.001$ & \\
\hline Region & & & & & & & $<0.001$ \\
\hline Asia & 8 & $189 / 291$ & $2.64(0.85,8.20)$ & 0.092 & $76.40 \%$ & $<0.001$ & \\
\hline Africa & 1 & 78/195 & $0.20(0.08,0.49)$ & $<0.001$ & / & / & \\
\hline GU vs. gastritis/FD & 3 & 93/139 & $3.55(0.71,17.77)$ & 0.123 & $76.00 \%$ & 0.016 & \\
\hline DU vs. gastritis/FD & 2 & $60 / 91$ & $3.15(0.28,35.66)$ & 0.354 & $87.10 \%$ & 0.005 & \\
\hline GC vs. gastritis/FD & 4 & $101 / 215$ & $2.09(0.51,8.66)$ & 0.308 & $79.10 \%$ & 0.002 & \\
\hline
\end{tabular}

The statistically significant results were highlighted in bold.

${ }^{\mathrm{a}}, \mathrm{P}$ value for heterogeneity test; ${ }^{\mathrm{b}}, \mathrm{P}$ value for Breslow-Day test.

Abbreviations: FD functional dyspepsia, PUD peptic ulcer disease, GU gastric ulcer, DU duodenal ulcer, GC gastric cancer.

region, a statistically increased risk for $\mathrm{GC}$ was observed in the America subgroup $(\mathrm{OR}=2.47,95 \%$ CI: 1.36, 4.51; $\mathrm{P}=0.003)$ and no significant association was found for Europe and Asia subgroups. However, result of BreslowDay test indicated no significant different effects of oipA "on" status on GC risk among different regions. Whereas in the subgroup analysis of detection method, only the association in immunblot subgroup reached significance $(\mathrm{OR}=$ 6.00, 95\% CI: 1.79, 20.15; P = 0.004), and the Breslow-Day test indicated a significant difference $(\mathrm{P}=0.034)$ among the sequencing/immunoblot subgroups in the ORs for oipA "on" status with GC risk.

\section{Association between oipA gene presence/absence and PUD and GC}

In the pooled estimate for PUD, the presence of oipA gene was not statistically associated with its overall risk compared with gastritis and FD controls $(\mathrm{OR}=1.93,95 \% \mathrm{CI}$ : $0.60,6.25 ; \mathrm{P}=0.278$, Table 2 and Figure 4). However, it demonstrated a significant heterogeneity among studies $\left(\mathrm{I}^{2}=85.70 \%, \mathrm{P}<0.001\right)$. To explore the source of heterogeneity, sensitivity analysis was performed. After the omitting of the most obvious outlier study with an OR of 90.39 (Figure 4) [14], significant heterogeneity still remained $\left(\mathrm{I}^{2}=84.8 \%, \mathrm{P}<0.001\right)$ and the conclusion did not change. 


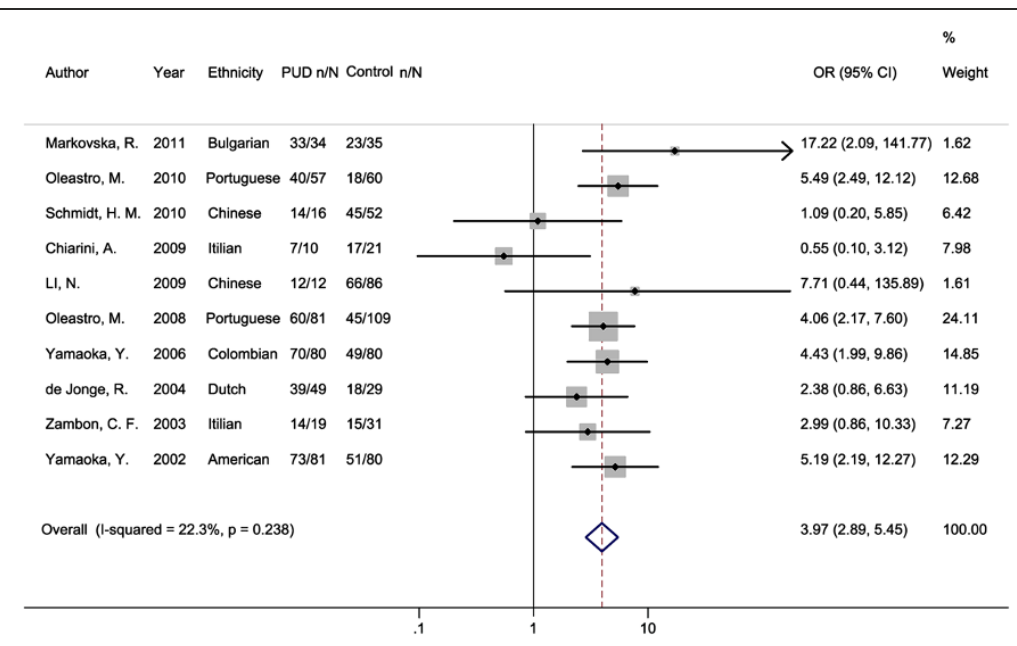

Figure 2 Forest plot for the association between oipA "on" status and PUD risk.

Subgroup differences and study design or quality also could not explain the source of heterogeneity. In subgroup analysis of different region, Africa subgroup analysis with only one article showed that the presence of oipA gene was statistically associated with a decreased risk of PUD compared with gastritis and FD controls $(\mathrm{OR}=0.20$, 95\% CI: 0.08, 0.49, P < 0.001). Moreover, the BreslowDay test did indicate a significant difference between Asia and Africa subgroups in the ORs for the presence of oipA gene $(\mathrm{P}<0.001)$. For DU and GU separately, the presence of oipA gene was also not related with the risks of both diseases.

In the pooled estimate for GC, we also observed no significant association of oipA gene positive with its overall risk compared with gastritis and FD controls $(\mathrm{OR}=2.09$, 95\% CI: 0.51, 8.66; P = 0.308, Table 2 and Figure 5). And meanwhile, significant heterogeneity was indicated among studies $\left(\mathrm{I}^{2}=79.1 \%, \mathrm{P}=0.002\right)$, thus sensitivity analysis was performed. The outlier study seemed to be the one carried out by Dabiri, H. et al. with an OR of 0.08 (Figure 5) [11]. After removing this data, the heterogeneity was no more significant $\left(\mathrm{I}^{2}=0.00 \%, \mathrm{P}=0.372\right)$, and the presence of oipA gene was associated with increased GC risk in the remaining studies $(\mathrm{OR}=4.11,95 \% \mathrm{CI}$ : 2.22, 7.62; $\mathrm{P}<0.001$ ). All the studies for GC risk used the PCRelectrophoresis method to detect the oipA presence/ absence status and the studied populations all came from Asian region. Therefore, no subgroup analysis was performed for the presence/ absence status of oipA gene with $\mathrm{GC}$ risk.

\section{Publication bias}

Funnel plots that qualitatively evaluated the publication bias of association between oipA presence/absence or on/off status and PUD were presented in Additional file 3: Figure S1. The Begg's test and Egger's test were performed to quantitatively evaluate the publication bias of the studies. No significant publication bias was observed

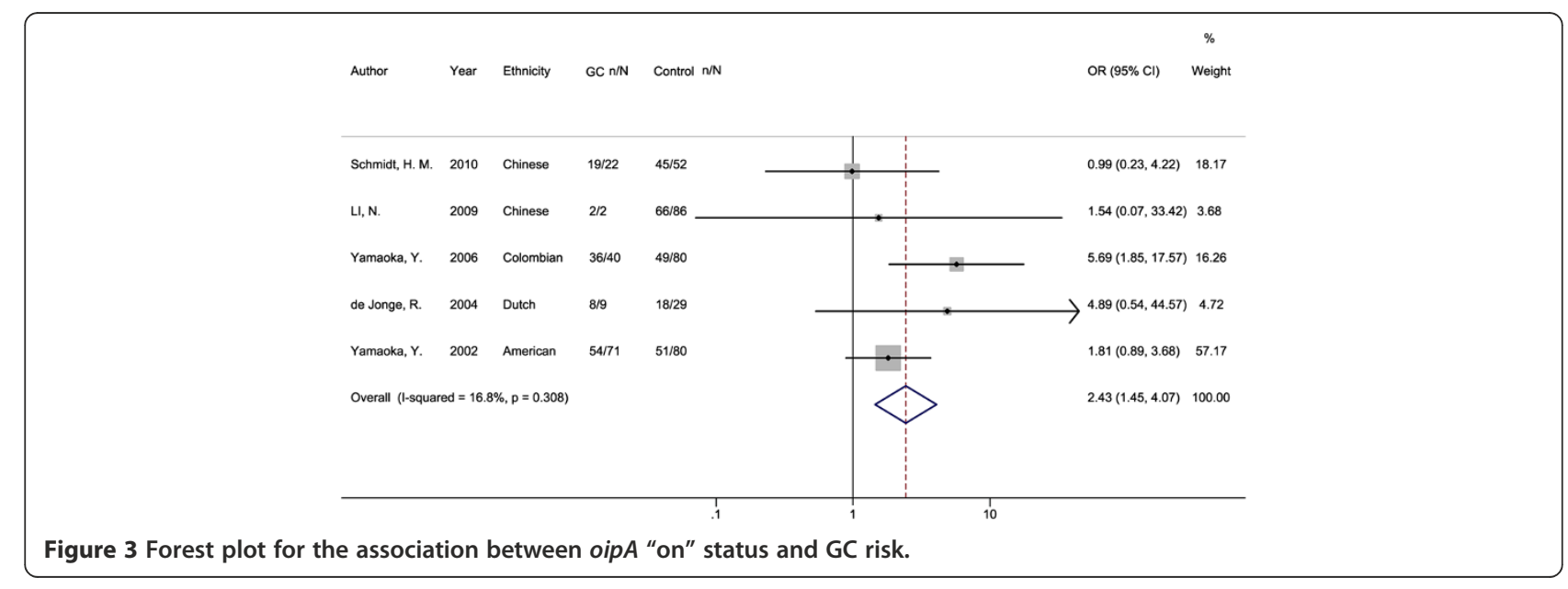


for meta-analyses of presence/absence or on/off patterns of oipA gene. The results for publication bias test were showed in Table 3.

\section{Discussion}

Results of studies regarding the relationship of $H$. pylori oipA gene with PUD and GC risks turn out to be controversial $[9,11,12,14,16-18,21]$. To our knowledge, this is the first meta-analysis evaluating the association between oipA gene status and PUD and GC. By performing the current meta-analysis, we found that when oipA gene exists, the oipA "on" status was associated with increased risks of PUD and GC compared with gastritis and FD controls. Null association was found between the presence of oipA gene and PUD or GC risks.

For the pooled analysis of oipA gene on/off status, we found association of oipA gene "on" status with increased overall risk of PUD compared with gastritis and FD controls, and no significant heterogeneity among studies was observed. Consistently, increased risks for PUD were observed in subgroup analyses although associations in Asia and GU subgroups did not reach statistical significance, which possibly due to small sample size of the PUD cases in Asia and GU subgroups (28 and 33 cases respectively). The potential influence of geographical region, age and detection method on the association strengthen between oipA "on" status and PUD risk was further clarified by homogeneity test. Observations indicated that children infected by $H$. pylori with oipA "on" status have a higher PUD risk than adults. One of the possible reasons explaining this phenomenon is that the gastric microenvironment of children may be more suitable for $H$. pylori with oipA "on" status to induce PUD. Another possible reason is the relatively low defense ability of children against pathogenic factor. Nevertheless, this different effect of oipA "on" status between children and adults was obtained with small groups of strains, which therefore requires future validation.

The pooled estimate also demonstrated that the oipA "on" status was associated with an increased risk of GC compared with gastritis and FD controls, and no significant heterogeneity among studies was observed. Subgroup

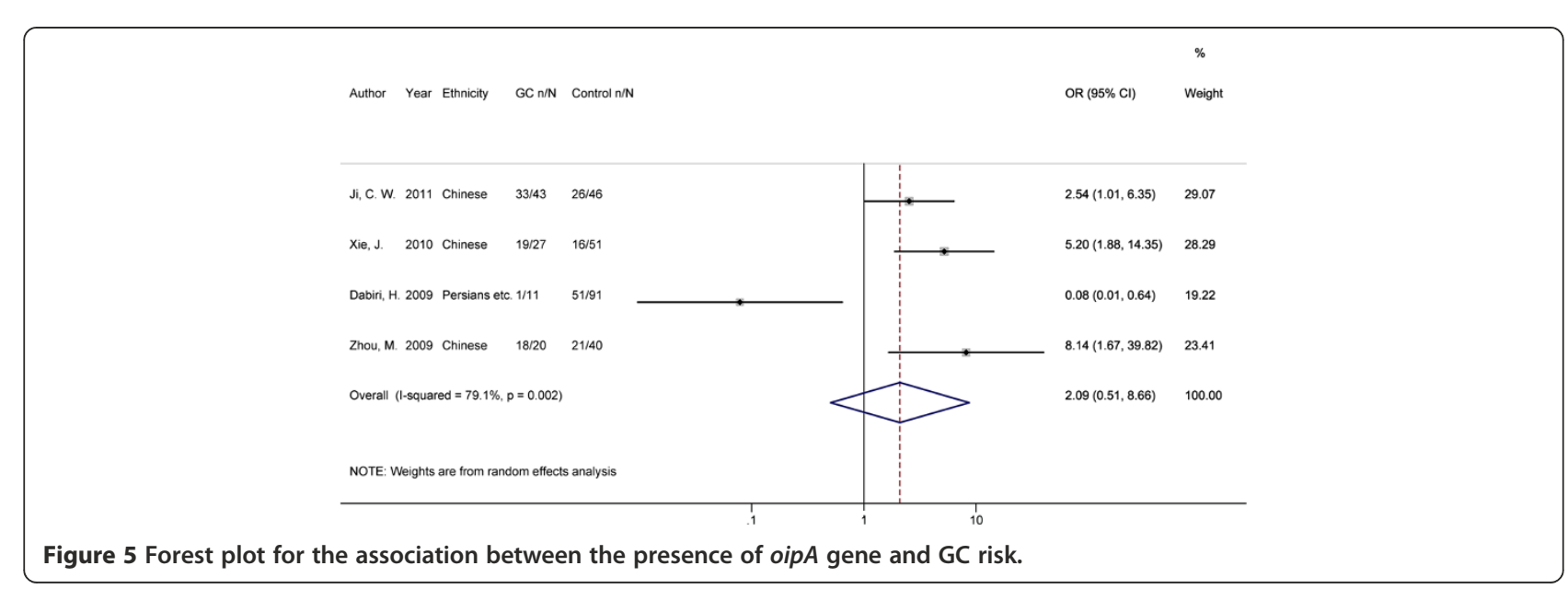


Table 3 Publication bias

\begin{tabular}{llllll}
\hline Variable & \multicolumn{2}{c}{ Begg's test } & & \multicolumn{2}{l}{ Egger's test } \\
\cline { 2 - 3 } & $\mathbf{z}$ value & $\mathbf{P ~ v a l u e ~}^{\mathbf{a}}$ & & $\mathbf{t}$ value & P value $^{\mathbf{a}}$ \\
\hline For oipA on/off status studies & & & \\
\hline PUD vs. gastritis/FD & -0.63 & 0.531 & -0.77 & 0.465 \\
\hline GC vs. gastritis/FD & 0.00 & 1.000 & 0.23 & 0.832 \\
\hline For oipA presence/absence studies & & & \\
\hline PUD vs. gastritis/FD & 1.05 & 0.293 & 1.64 & 0.163 \\
\hline GC vs. gastritis/FD & -0.68 & 0.497 & -0.93 & 0.452 \\
\hline
\end{tabular}

${ }^{a}$, $\mathrm{P}$ value $<0.1$ was considered as significant publication bias. Abbreviations: FD functional dyspepsia, PUD peptic ulcer disease, GC gastric cancer.

analyses indicated that oipA "on" status showed a consistent tendency toward increasing the risk of GC development although Europe, Asia, PCR-sequencing subgroups did not reach statistical significance. However, the test for homogeneities between subgroups indicated no significant difference for Europe/Asia/America subgroups. Statistical different effect was only indicated between PCR-sequencing and immunoblot subgroups. Although the PCR-based sequencing determines the functional status of oipA gene by detecting the number of CT repeats in its signal-peptide region, this method could not guarantee the expression of OipA protein. The high degree of genetic diversity of oipA gene may also complicate the interpretation of PCR based methods and may possibly result in an underestimation of the frequency of the functional status of the oipA gene.

Some mechanism studies may partially explain the association between oipA "on" status with PUD and GC. In 2000, by gene knockout models, Yamaoka et al. initially linked oipA gene "on" status with increased IL-8 production in gastric cancer cells [6]. Similar effects were detected in Straubinger et al's study using cat models [31]. Subsequently, by challenging the volunteers with a cagA negative, oipA functional "on" strain of H. pylori, Graham et al. confirmed the role of oipA "on" status in inducing IL-8 levels in human. This author reported that the IL-8 levels in gastric mucosa increased even up to 20-fold by two weeks after inoculation [32]. Yamaoka et al. further unraveled that OipA was necessary for full activation of the IL-8 promoter and acted via the STAT1-IRF1-ISRE pathway [33]. Importantly, the IL-8 is one of the most essential proinflammatory factors, which acts as a potent chemoattractant and activator of neutrophils [34]. It has been suggested that IL- 8 is closely linked with the tumorigenesis, angiogenesis and intracellular adhesion of cancer [35]. Therefore, it is tempting to speculate that the association of oipA "on" status with increased PUD and GC risks may be, at least in part, explained by a role in inducing IL-8 secretion.

It is worth noting that the detection of the presence/ absence of oipA gene could not reflect the specific functional status of this gene, since the signal-peptide region of oipA gene varies significantly among $H$. pylori strains. Merely assessing the presence/absence of oipA gene would result in an underestimation of the influence of the functional status of oipA gene on PUD and GC development. Therefore, more caution should be taken when link the presence of oipA gene with phenotypic risk regardless of its functional status. The investigators that only examined the presence/ absence of oipA gene may overlook the importance of the on/off status of oipA or could not perform the sequencing. The synthesis of the data in the present meta-analysis also suggested that only studying the "presence/absence" of oipA gene provided insufficient evidence to investigate the exact role of $H$. pylori oipA gene in gastrointestinal diseases. Normally, investigators should sequence oipA gene and take the functional on/off status of oipA into consideration.

The prevention and treatment for PUD and GC cause significant financial burdens around the world [36,37]. Because $H$. pylori is one important cause for PUD and GC, the identification of specific type of $H$. pylori isolates associated with PUD and GC risks would significantly reduce the costs of the screening and prevention for PUD and GC. The OR for PUD was found to be higher than that of GC (3.97 vs. 2.43) in this meta-analysis. The predictive role of oipA functional status in risks of PUD and GC may differ because of their distinct pathogenic mechanisms. However, we could not definitely conclude that the oipA "on" status is more closely linked with PUD than GC by numerically judging the OR values based on the currently-limited study samples. It is anticipated that oipA "on" status would be a promising indicator for $H$. pylori infected patients with increased risk for PUD or $\mathrm{GC}$ in the future.

We are aware that this meta-analysis has its own limitations. First, only articles in English or Chinese were selected. And we searched four databases without referring to other databases like EMBASE, which may result in selection bias. Second, the quality assessment of NOS indicated that most studies were at an intermediate level of quality mainly due to not matching for age or gender. In addition, the control group mixed the gastritis and FD, and some studies did not clearly describe that the control group excluded other kinds of diseases, which may result in an underestimation of the effect of oipA gene. Third, the sample size is not sufficiently large which may partially due to the limitation of the present laboratory technique for the isolation and cultivation of $H$. pylori isolates. Besides, numbers of included studies were relatively small, so the power for publication bias test was relatively low. Fourth, most studies were from Asia, thus the generalizability of our conclusion was limited. Fifth, significant heterogeneities were indicated for some comparisons particularly in presence/absence analysis, which could not be explained 
by subgroup analyses or sensitivity analyses. Moreover, the limited number of included studies precluded us from performing meta-regression to further explore the source of heterogeneity. Sixth, other important data such as age, gender, family history, status of other virulence factors and environment factors were not available to investigate the interaction between oipA gene status and these factors. The combined effect of oipA and other virulent factors should also be studied in future study.

\section{Conclusions}

To be concluded, when oipA exists, the functional "on" status of this gene showed association with increased risks for PUD and GC compared with gastritis and FD controls. However, merely investigating the presence/absence of oipA would overlook the importance of its functional on/ off status and would not be reliable to predict risks of PUD and GC. Further large-scale and well-designed studies concerning on/off status of oipA are required to confirm our meta-analysis results.

\section{Additional files}

\section{Additional file 1: PRISMA checklist. \\ Additional file 2: Table S1. Primers of PCR for oipA gene presence/ absence and on/off status detection. Table S2. Detailed information of the included studies. Table S3. Results of Newcastle - Ottawa scale (NOS) assessment for the included studies.}

Additional file 3: Figure S1. Funnel plots of oipA gene presence/ absence and oipA gene on/off status and PUD A, funnel plot for studies of association between oipA gene presence/absence and PUD; B, funnel plot for studies of association between oipA gene on/off status and PUD.

\section{Competing interests}

The authors declare that they have no competing interests.

\section{Authors' contributions}

$\mathrm{J}$ and $\mathrm{CH}$ performed statistical analysis, data interpretation and wrote the paper. MC and ZW analyzed the data and revised the manuscript. CX and YY conceived and designed this study and revised the manuscript. All authors read and approved the final manuscript.

\section{Funding}

This study is supported by grants from National Basic Research Program of China (973 Program Ref No.2010CB529304), the grants of the Science and Technology Project of Liaoning province (Ref No.2011225002) and the grants of the Science and Technology Project of Liaoning province (Ref No.2012225016).

Received: 19 March 2013 Accepted: 18 October 2013

Published: 20 November 2013

\section{References}

1. Yamaoka Y: Pathogenesis of Helicobacter pylori-related gastroduodenal diseases from molecular epidemiological studies. Gastroenterol Res Pract 2012, 2012:371503

2. Algood HM, Cover TL: Helicobacter pylori persistence: an overview of interactions between $\mathrm{H}$. pylori and host immune defenses. Clin Microbiol Rev 2006, 19(4):597-613.

3. Delahay RM, Rugge M: Pathogenesis of Helicobacter pylori infection. Helicobacter 2012, 17(Suppl 1):9-15.

4. Yamaoka Y: Mechanisms of disease: Helicobacter pylori virulence factors. Nat Rev Gastroenterol Hepatol 2010, 7(11):629-641.
5. Basso D, Plebani M, Kusters JG: Pathogenesis of Helicobacter pylori infection. Helicobacter 2010, 15(Suppl 1):14-20.

6. Yamaoka Y, Kwon DH, Graham DY: A M(r) 34,000 proinflammatory outer membrane protein (oipA) of Helicobacter pylori. Proc Natl Acad Sci USA 2000, 97(13):7533-7538.

7. Ando T, Peek RM, Pride D, Levine SM, Takata T, Lee YC, Kusugami K, van der Ende A, Kuipers EJ, Kusters JG, et al: Polymorphisms of Helicobacter pylori HP0638 reflect geographic origin and correlate with cagA status. J Clin Microbiol 2002, 40(1):239-246.

8. Ji CW, Yang L: Relationship between Helicobacter pylori oipA gene and the expression of IL-17 in gastric mucosa. Med J Chin PLA 2011, 36(3):228-230

9. Ben Mansour K, Fendri C, Zribi M, Masmoudi A, Labbene M, Fillali A, Ben Mami N, Najjar T, Meherzi A, Sfar T, et al: Prevalence of Helicobacter pylori vacA, cagA, iceA and oipA genotypes in Tunisian patients. Ann Clin Microbiol Antimicrob 2010, 9:10.

10. Xie J, Yang L: Distribution of cagA Genotype, oipA Genotype, cagX Genotype of Helicobacter Pylori in Ningxia and the Relationship with Chronic Gastrointestinal Disease and Gastric Cancer; 2010.

11. Dabiri H, Maleknejad P, Yamaoka Y, Feizabadi MM, Jafari F, Rezadehbashi M, Nakhjavani FA, Mirsalehian A, Zali MR: Distribution of Helicobacter pylori cagA, cagE, oipA and vacA in different major ethnic groups in Tehran, Iran. J Gastroenterol Hepatol 2009, 24(8):1380-1386.

12. Zhou M: The relationship between oipa protein of Helicobacter pylori and HPassociated diseases; 2009

13. Salih BA, Abasiyanik MF, Ahmed N: A preliminary study on the genetic profile of cag pathogenicity-island and other virulent gene loci of Helicobacter pylori strains from Turkey. Infect Genet Evol 2007, 7(4):509-512.

14. Zhang J, Yu FF, Chen YX, Chen H: Detection of cagA, oipA and iceA1 genes of Helicobacter pylori and its significance. Chin J Health Lab Technol 2004, 14(2):133-134.

15. Markovska R, Boyanova L, Yordanov D, Gergova G, Mitov I: Helicobacter pylori oipA genetic diversity and its associations with both disease and cagA, vacA s, m, and i alleles among Bulgarian patients. Diagn Microbiol Infect Dis 2011, 71(4):335-340.

16. Oleastro M, Santos A, Cordeiro R, Nunes B, Megraud F, Menard A: Clinical relevance and diversity of two homologous genes encoding glycosyltransferases in Helicobacter pylori. J Clin Microbiol 2010, 48(8):2885-2891.

17. Schmidt HM, Andres S, Nilsson C, Kovach Z, Kaakoush NO, Engstrand L, Goh KL, Fock KM, Forman D, Mitchell H: The cag PAl is intact and functional but HP0521 varies significantly in Helicobacter pylori isolates from Malaysia and Singapore. Eur J Clin Microbiol Infect Dis 2010, 29(4):439-451.

18. Chiarini A, Cala C, Bonura C, Gullo A, Giuliana G, Peralta S, D'Arpa F, Giammanco A: Prevalence of virulence-associated genotypes of Helicobacter pylori and correlation with severity of gastric pathology in patients from western Sicily, Italy. Eur J Clin Microbiol Infect Dis 2009, 28(5):437-446

19. Li N, She FF, Lin $\mathrm{X}$, Che H: Helicobacter pylori oipA gene switch status and its association with gastic diseases. Chin J Dig 2009, 29(2):122-125.

20. Oleastro M, Cordeiro R, Ferrand J, Nunes B, Lehours P, Carvalho-Oliveira I Mendes Al, Penque D, Monteiro L, Megraud F, et al: Evaluation of the clinical significance of homB, a novel candidate marker of Helicobacter pylori strains associated with peptic ulcer disease. J Infect Dis 2008 198(9):1379-1387.

21. Yamaoka Y, Ojo O, Fujimoto S, Odenbreit S, Haas R, Gutierrez O, El-Zimaity HM, Reddy R, Arnqvist A, Graham DY: Helicobacter pylori outer membrane proteins and gastroduodenal disease. Gut 2006, 55(6):775-781.

22. De Jonge R, Pot RG, Loffeld RJ, Van Vliet AH, Kuipers EJ, Kusters JG: The functional status of the Helicobacter pylori sabB adhesin gene as a putative marker for disease outcome. Helicobacter 2004, 9(2):158-164.

23. Zambon CF, Navaglia F, Basso D, Rugge M, Plebani M: Helicobacter pylori babA2, cagA, and s1 vacA genes work synergistically in causing intestinal metaplasia. J Clin Pathol 2003, 56(4):287-291.

24. Yamaoka Y, Kikuchi S, El-Zimaity HM, Gutierrez O, Osato MS, Graham DY: Importance of Helicobacter pylori oipA in clinical presentation, gastric inflammation, and mucosal interleukin 8 production. Gastroenterology 2002, 123(2):414-424.

25. Stang A: Critical evaluation of the Newcastle-Ottawa scale for the assessment of the quality of nonrandomized studies in meta-analyses. Eur J Epidemiol 2010, 25(9):603-605. 
26. Higgins JP, Thompson SG: Quantifying heterogeneity in a meta-analysis. Stat Med 2002, 21(11):1539-1558.

27. Mantel N, Haenszel W: Statistical aspects of the analysis of data from retrospective studies of disease. J Natl Cancer Inst 1959, 22(4):719-748.

28. DerSimonian R, Laird N: Meta-analysis in clinical trials. Control Clin Trials 1986, 7(3):177-188

29. Begg CB, Mazumdar M: Operating characteristics of a rank correlation test for publication bias. Biometrics 1994, 50(4):1088-1101.

30. Egger M, Davey Smith G, Schneider M, Minder C: Bias in meta-analysis detected by a simple, graphical test. BMJ 1997, 315(7109):629-634

31. Straubinger RK, Greiter A, McDonough SP, Gerold A, Scanziani E, Soldati S, Dailidiene D, Dailide G, Berg DE, Simpson KW: Quantitative evaluation of inflammatory and immune responses in the early stages of chronic Helicobacter pylori infection. Infect Immun 2003, 71(5):2693-2703.

32. Graham DY, Opekun AR, Osato MS, El-Zimaity HM, Lee CK, Yamaoka Y, Qureshi WA, Cadoz M, Monath TP: Challenge model for Helicobacter pylori infection in human volunteers. Gut 2004, 53(9):1235-1243.

33. Yamaoka Y, Kudo T, Lu H, Casola A, Brasier AR, Graham DY: Role of interferon-stimulated responsive element-like element in interleukin-8 promoter in Helicobacter pylori infection. Gastroenterology 2004, 126(4):1030-1043.

34. Remick DG: Interleukin-8. Crit Care Med 2005, 33(12 Suppl):S466-S467.

35. Waugh DJ, Wilson C: The interleukin-8 pathway in cancer. Clin Cancer Res 2008, 14(21):6735-6741.

36. Yuan Y, Padol IT, Hunt RH: Peptic ulcer disease today. Nat Clin Pract Gastroenterol Hepatol 2006, 3(2):80-89.

37. Thun MJ, DeLancey JO, Center MM, Jemal A, Ward EM: The global burden of cancer: priorities for prevention. Carcinogenesis 2010, 31(1):100-110.

doi:10.1186/1471-2334-13-555

Cite this article as: Liu et al:: Association of presence/absence and on/ off patterns of Helicobacter pylori oipA gene with peptic ulcer disease and gastric cancer risks: a meta-analysis. BMC Infectious Diseases 2013 13:555

\section{Submit your next manuscript to BioMed Central and take full advantage of:}

- Convenient online submission

- Thorough peer review

- No space constraints or color figure charges

- Immediate publication on acceptance

- Inclusion in PubMed, CAS, Scopus and Google Scholar

- Research which is freely available for redistribution 